\title{
Probiotic-associated high-titer anti-B in a group A platelet donor as a cause of severe hemolytic transfusion reactions
}

\author{
Jennifer Daniel-Johnson, Susan Leitman, Harvey Klein, Harvey Alter, Agnes Lee-Stroka, \\ Phillip Scheinberg, Jeremy Pantin, and Karen Quillen \\ Department of Transfusion Medicine, Warren G. Magnuson Clinical Center, and the Hematology \\ Branch, National Heart, Lung, and Blood Institute, National Institutes of Health, Bethesda, \\ Maryland
}

\section{Abstract}

BACKGROUND-Hemolytic transfusion reactions (HTRs) can occur with transfusion of platelets (PLTs) containing ABO-incompatible plasma. Reported cases have involved group O donors. Two cases of PLT-mediated HTRs associated with the same group A plateletpheresis component, collected from a donor taking high doses of probiotics are reported.

CASE REPORT-Case 1 was a 40 -year-old $69-\mathrm{kg}$ group B stem cell transplant patient who received one-half of a group A plateletpheresis component. Severe back pain occurred 10 minutes into the transfusion, accompanied by anemia and hyperbilirubinemia. Case 2 was a 5-year-old 26$\mathrm{kg}$ group B male with aplastic anemia who received the other half of the same plateletpheresis component, volume reduced to $37 \mathrm{~mL}$. Syncope occurred immediately after the transfusion, with laboratory evidence of hemolysis a few hours later.

RESULTS-Serologic investigation of posttransfusion samples from both patients revealed positive direct anti-globulin tests: C3d only for Case 1 and immunoglobulin (Ig)G and C3d for Case 2; the eluates contained anti-B. The group A donor's anti-B titer was 16,384 at saline and IgG phases. Donor lookback revealed that the donor had donated 134 apheresis PLTs over many years. For 3 years, he had intermittently taken probiotics; 3 weeks before the index donation, he began taking three tablets of probiotics every day. Lookback of prior group B recipients uncovered a case of acute hemolysis that was not recognized at the time. The solubilized probiotic inhibited anti-B in vitro.

CONCLUSION-Non-group O PLT donors can have high-titer anti-A or anti-B that might mediate HTRs, and probiotic ingestion in blood donors represents a novel mechanism of stimulating high-titer anti-B.

Passively mediated hemolytic transfusion reactions (PMHTRs) occur with minor ABOincompatible platelet (PLT) transfusions when the donor plasma contains high-titer anti-A or anti-B or when a large amount of incompatible plasma is transfused over a short period of time. Recipients with a small blood volume such as pediatric patients are particularly at risk. To date, all reported cases of PMHTR have involved group O donors, whose plasma commonly contains anti-A and anti-B with hemolytic potential. ${ }^{1,2}$ We report two group B recipients who developed PMHTR from the same group A apheresis PLT component,

Address reprint requests to: Karen Quillen, 88 East Newton Street, H-3600, Boston, MA 02118; kq@ bu.edu. CONFLICT OF INTEREST

The authors have no conflict of interest to disclose. The views expressed in this paper are those of the authors and are not to be construed as the official position of the United States Department of Health and Human Services. 
collected from a donor who had recently started taking high doses of a commercially available probiotic.

\section{CASE REPORT}

\section{Case 1}

A 40-year-old 69-kg man who had recently undergone a second peripheral blood stem cell transplant from the same group B D+ matched sibling donor for relapsed acute myeloid leukemia had persistent thrombocytopenia 1 month posttransplant. He was scheduled for 6 units of PLTs (one-half of a double-apheresis PLT collection). Fifteen minutes and $100 \mathrm{~mL}$ into the transfusion, he developed severe back and flank pain associated with hypertension (blood pressure 112/72 to 150/92) and chest pain. The transfusion was discontinued; a posttransfusion specimen was noted to be grossly hemolyzed and the urine specimen was dark brown. The hemoglobin $(\mathrm{Hb})$ level had decreased from $9.7 \mathrm{~g} / \mathrm{dL}$ pretransfusion to 5.5 $\mathrm{g} / \mathrm{dL}$. The pre- and posttransfusion laboratory results are shown in Fig. 1. The patient was treated supportively with intravenous hydration and transfusion of 3 units of red blood cells (RBCs). Cardiac and renal function remained stable, and the patient was discharged 4 days later. When the other half of the apheresis PLT component was traced, it was discovered to have been administered 2 hours earlier to another patient (Case 2 below).

\section{Case 2}

A 5-year-old 26-kg boy with severe aplastic anemia 3 months postimmunosuppressive therapy had received the second half of the implicated apheresis PLTs. The component had been volume reduced (to $37 \mathrm{~mL}$ ) in accordance with institutional policy for out-of-group PLTs to pediatric patients. At the completion of the transfusion, the patient had a witnessed syncopal episode after defecating. He remained minimally responsive for 1 minute and very drowsy thereafter. Computed tomography of the head did not show any intracranial hemorrhage. By the time of the index transfusion reaction (Case 1 above), the second patient had improved clinically and was awake and alert without any complaints. The pretransfusion and posttransfusion laboratory results are shown in Fig. 2. The patient had a nadir $\mathrm{Hb}$ level of $7.1 \mathrm{~g} / \mathrm{dL} 9$ hours after PLT transfusion (a decrease of $2.3 \mathrm{~g} / \mathrm{dL}$ ), received one RBC transfusion, and was discharged home 3 days later.

\section{MATERIALS AND METHODS}

\section{Transfusion reaction investigation and donor anti-B titers}

The initial workup of the transfusion reactions consisted of a clerical check, visual inspection of the centrifuged specimen, and direct antiglobulin test (DAT). Plasma from the donor of the implicated unit was assayed for anti-B titers along with male and female controls of the same ABO group. Serial twofold dilutions were performed. After the addition of group B RBCs, each dilution was tested after 30-minute incubation at room temperature (RT) and 30-minute incubation at $37^{\circ} \mathrm{C}$ followed by antiglobulin (immunoglobulin [Ig]G) testing. The titer was expressed as the reciprocal of the highest dilution that showed macroscopic agglutination. Stored plasma samples obtained from the implicated donor for another study within the past 3 years were retrieved and titrated.

\section{Inhibition studies with probiotic formula}

Problems were encountered in getting the probiotic (Primal Defense HSO Probiotic Formula, Garden of Life, West Palm Beach, FL) into solution. When a $1 \mathrm{mg} / \mathrm{mL}$ suspension in $\mathrm{pH} 7.4$ phosphate-buffered saline (PBS) was used, it did not inhibit anti-B; it was suspected that the probiotic had not gone into solution. More concentrations of probiotic were tested. A suspension of $3 \mathrm{~g} / 60 \mathrm{~mL}(50 \mathrm{mg} / \mathrm{mL})$ was boiled for 10 minutes and 
centrifuged; the supernatant now inhibited anti-B (see Results). Equal amounts of polyclonal anti-B, from another donor source, probiotic, and a control substituting PBS for probiotic, were incubated for 1 hour. Serial dilutions were prepared with either the anti-B plus probiotic or the anti-B plus PBS control. One drop of group B RBCs was added to each tube and incubated for 30 minutes at RT. The titer was expressed as the highest dilution at which inhibition occurred.

\section{RESULTS}

The pretransfusion specimen from Patient 1 typed as group B D+ and had a negative DAT; the serum showed no evidence of hemolysis. The patient's posttransfusion sample showed severe hemolysis with brown plasma and a DAT positive for C3d. The RBCs now reacted with anti-A and anti-B, but a saline control was also positive. When the RBCs were treated with dithiothreitol to prevent RBC-bound IgM interfering, the RBCs no longer reacted with anti-A and the saline control was negative. The patient's serum reacted with group A and B RBCs, but not group O RBCs. The patient's untreated RBCs were nonreactive on a lectin panel (data not shown), ruling out polyagglutination. An eluate demonstrated anti-B.

Subsequent postreaction specimens obtained the following day(s) did not demonstrate any $\mathrm{ABO}$ discrepancy. The urine was grossly dark brown, had $3+\mathrm{Hb}$ on dipstick analysis, and had no RBCs on microscopic examination.

The pretransfusion specimen from Patient 2 typed as group B D+ and had a negative DAT; the serum was not hemolyzed. The posttransfusion specimen was grossly hemolyzed and typed as group B D+; a DAT was positive for both $\mathrm{IgG}$ and C3d. An eluate demonstrated anti-B. Urinalysis was $1+$ positive for Hb without RBCs on microscopic examination. Of note, for both patients, the degree of hemolysis interfered with routine chemistry testing, and the results for several analytes were only obtainable using a sodium lithium collection tube.

\section{Donor serologic investigation and lookback}

The donor was group A D+ with a negative antibody screen; anti-B titers were 16,384 at both RT (saline) and IgG phases on the ethylenediaminetetraacetate specimen drawn at the time of the implicated PLT collection for type and screen. The control titers ranged from 16 to 64 in the saline phase and 16 to 128 at the $\operatorname{IgG}$ phase. Donor plasma samples from four prior donations were available for testing. The anti-B titers were 8192 at 3 years and 2 years before this donation and 4096 on two occasions 2 months before this donation.

The donor was a 69-year-old male who had donated 136 units of whole blood and 134 units of apheresis PLTs since 1977. He was in good health. His medications were $20 \mathrm{mg} / \mathrm{day}$ omeprazole, $0.8 \mathrm{mg} /$ day folate, omega 3 fatty acids/fish oil 3 tablets/day, $500 \mathrm{mg} / \mathrm{day}$ magnesium salts, and three tablets of a probiotic per day. He had started this particular probiotic supplement 3 weeks before this donation, but had intermittently taken lowerpotency probiotics for 3 years. The current probiotic contains seven strains of lactobacilli, three strains of bifidobacteria, and Bacillus subtilis.

A sample of the probiotic was obtained from the donor and used for inhibition studies. The titer of anti-B in a group A plasma (from a randomly selected PLT donor) was reduced threefold, from 64 to 8 , after incubation at RT with a solubilized suspension of the probiotic tablets.

Lookback on prior donations by this donor over an 18-year period showed that only two prior components had been transfused to group B recipients. The first recipient was a 41year-old group B D+ woman with severe aplastic anemia who received apheresis PLTs from the implicated donor without any evidence of an acute transfusion reaction on one outpatient 
visit. Routine laboratory testing performed 4 days later noted a $1.6 \mathrm{~g} / \mathrm{dL}$ decrease in $\mathrm{Hb}$ with a slightly elevated lactate dehydrogenase. The patient was asymptomatic, and hemolysis was not suspected at the time. The second recipient was a 53-year-old group B D+ woman with non-Hodgkin's lymphoma undergoing chemotherapy, complicated by Clostridium difficile colitis and septic shock, who received apheresis PLTs from the implicated donor. The next day, she deteriorated clinically with lactic acidosis and worsening anemia $(\mathrm{Hb} 4.3 \mathrm{~g} / \mathrm{dL}$, from $8.3 \mathrm{~g} / \mathrm{dL}$ ). An abdominal catastrophe was suspected, and she underwent an exploratory laparotomy, subtotal colectomy, and partial small bowel resection for bowel ischemia. No significant intraabdominal bleeding was noted, and at the time hemolysis related to transfusion was not suspected. Laboratory measures before and after the PLT transfusion were consistent, in retrospect, with severe hemolysis. The patient had a prolonged hospital course as a result of multiple postoperative infections.

\section{DISCUSSION}

To our knowledge, these cases represent the first reported cases of hemolytic transfusion reactions (HTRs) associated with a group A apheresis PLT donor. Although the true incidence of PMHTR is not known, the range is 1:1000 to 1:25,000 for all PLT transfusions and 1:100 to 1:9000 for minor-incompatible PLT transfusions. ${ }^{1}$ In all prior reports implicated donors were group $\mathrm{O}$, and recipients were usually group $\mathrm{A}$ with the exception of a few cases involving group $\mathrm{AB}$ and group $\mathrm{B}$ recipients. ${ }^{1,2}$ The clinical presentations and severity of these reactions vary widely. In one recent review ${ }^{1}$ summarizing 28 cases of PMHTR, $82 \%$ of the PLTs implicated were apheresis PLTs, the mean volume of minorincompatible plasma infused was $350 \mathrm{~mL}$, the mean saline anti-A/B titer was 2403 (range, 128 to 16,384), and the mean decrease in $\mathrm{Hb}$ was $5.8 \mathrm{~g} / \mathrm{dL}$. Our second index case and lookback cases underscore the fact that PMHTR can be underrecognized especially in patients receiving outpatient transfusion.

In most cases of PMHTR the cause of the high-titer anti-A or anti-B in the donor is unknown. ${ }^{3}$ Known causes include pregnancy, recent vaccination, and ingestion of high-dose live bacteria. Immunization with pneumococcal vaccine ${ }^{4}$ and certain toxoids ${ }^{5}$ including tetanus and diphtheria was shown to cause a marked rise in isoagglutinins titers, especially anti-A, attributable to A-like substance in the polysaccharide capsule of Streptococcus pneumoniae, and the presence of A-like substance in hog stomach pepsin used in toxoid production. It is not known how long these high titers persist after vaccination.

The implicated donor's high-titer anti-B was associated with probiotic ingestion. Probiotics are dietary supplements that contain live bacteria and exert their putative benefits by preventing bowel colonization, adhesion, and invasion by pathogenic organisms and by modulating host immune response. Among healthy individuals, they are promoted to alleviate the symptoms of lactose intolerance, antibiotic-associated gastrointestinal side effects, allergy manifestations, and the common cold. ${ }^{6,7}$ Our inhibition studies demonstrate that the bacteria in some probiotic formulations contain B-like antigens that may stimulate increased production of anti-B when ingested by subjects lacking the corresponding antigen. We postulate that our donor's exposure to high doses of probiotic stimulated the formation of high-titer anti-B, although we do not have stored plasma that dates back more than 3 years to test our hypothesis.

The formation of anti-A and anti-B is known to result from exposure to bacterial antigens that are similar in structure to the A and B blood group antigens as the gut becomes colonized in early infancy. These antibodies develop after the first 4 to 6 months of life. Most of the bacteria expressing A, B, and $\mathrm{H}$ substance are ubiquitous Gram-negative bacteria that eventually colonize the normal gut. For example, Escherichia coli serotype 
086:B7 has a B-like antigen on its lipopolysaccharide chain that can stimulate anti-B production. Springer and Horton ${ }^{8}$ exposed subjects to $E$. coli $086: B 7$, either by ingestion or by inhalation, and measured pre- and postexposure anti-B titers. One-third of healthy adults had a significant increase in the titer of anti-B, and the anti-B response was strongest in those who produced high-titer antibodies to E. coli.

In addition to directly stimulating anti-B production, probiotics may nonspecifically enhance humoral immunity. In one study, lactobacillus administered to children with acute gastroenteritis increased IgG, IgA, and IgM levels; ${ }^{9}$ in another study, probiotics given to infants to prevent atopy improved antibody response to Haemophilus influenzae Type b vaccination. ${ }^{10}$

Various strategies have been proposed to reduce the risk of a PMHTR (Table 1). Screening for high-titer anti-A, anti-B, or anti-A,B has been in place in the United Kingdom since 2002, although donor screening using a titer cutoff of 100 on an automated platform has not eliminated PMHTR; ${ }^{11}$ a recent study using a standardized method of titration still showed variable titer results highlighting the difficulty in setting a reproducible cutoff titer. ${ }^{12}$ Other strategies include volume reducing or washing minor-incompatible PLTs, limiting the amount of incompatible plasma transfused over 24 hours, the use of PLT storage media that replaces much of the donor plasma, and transfusion of whole blood-derived PLT concentrates in lieu of apheresis PLTs. ${ }^{1,13}$ Many of these methods will not prevent a PMHTR if very-high-titer anti-A or anti-B is present. A volume as small as 30 to $50 \mathrm{~mL}$ can cause a PMHTR whether derived from 1 unit of pooled PLTs, the residual volume of plasma in a volume reduced apheresis component, or even PLTs stored in additive storage media. In our second case, $37 \mathrm{~mL}$ of residual plasma in a volume-reduced product was sufficient to cause hemolysis. In another report two group A patients had a PMHTR when transfused with "dry PLTs" resuspended in additive storage media. ${ }^{14}$ A recent report also found anti-A and anti-B titers in pooled PLTs to be comparable to those of apheresis PLTs. ${ }^{15}$

The incidental finding of an ABO discrepancy on the first postreaction specimen in Case 1 was unexpected. We speculate that the patient's RBCs were so heavily coated with passive anti-B that they spontaneously agglutinated on initial testing. Such spontaneous in vitro agglutination has been described in the setting of passively acquired high-titer anti-B. ${ }^{16}$ The initial reactivity with reverse grouping B cells was similarly attributable to passively acquired anti-B from donor plasma.

In conclusion, we have demonstrated that non-group O PLT donors can have high-titer antiB that can mediate HTRs, that PMHTR may be underrecognized, and that probiotic ingestion represents a novel mechanism of stimulating high-titer anti-B. The effects of probiotic ingestion on anti-B production in otherwise healthy adults and their clinical significance merit further investigation in a prospective study. We are currently investigating this to determine if donors should be questioned about probiotic consumption and to guide management of donors who admit to probiotic consumption.

\section{ABBREVIATIONS}

$\begin{array}{ll}\text { HTR(s) } & \text { hemolytic transfusion reaction(s) } \\ \text { PMHTR(s) } & \text { passively mediated hemolytic transfusion reaction(s) } \\ \text { RT } & \text { room temperature }\end{array}$




\section{References}

1. Cooling L. ABO and platelet transfusion therapy. Immuno-hematology. 2007; 23:20-33.

2. Fung MK, Downes KA, Shulman I. Transfusion of platelets containing ABO-incompatible plasma. Arch Pathol Lab Med. 2007; 131:909-16. [PubMed: 17550318]

3. Larsson LG, Welsh VJ, Ladd DJ. Acute intravascular hemolysis secondary to out-of-group platelet transfusion. Transfusion. 2000; 40:902-6. [PubMed: 10960514]

4. Boyer KM, Theeravuthichai J, Vogel LC, Orlina A, Gotoff SP. Antibody response to group B streptococcus type III and AB blood group antigens induced by pneumococcus vaccine. J Pediatr. 1981; 98:374-8. [PubMed: 7009817]

5. Elliott GB. Transiently dangerous universal blood donor. Can Med Assoc J. 1954; 70:571-4. [PubMed: 13160893]

6. Doron S, Gorbach SL. Probiotics: their role in the treatment and prevention of disease. Expert Rev Anti Infect Ther. 2006; 4:261-75. [PubMed: 16597207]

7. De Vrese M, Winkler P, Rautenberg P, Harder T, Noah C, Laue C, Ott S, Hampe J, Schreiber S, Heller K, Schrezenmeir J. Probiotic bacteria reduced duration and severity but not the incidence of common cold episodes in a double blind, randomized, controlled trial. Vaccine. 2006; 24:6670-4. [PubMed: 16844267]

8. Springer GF, Horton RE. Blood group isoantibody stimulation in man by feeding blood groupactive bacteria. J Clin Invest. 1969; 48:1280-91. [PubMed: 4893685]

9. Kaila M, Isolauri E, Soppi E, Virtanen E, Laine S, Arvilommi H. Enhancement of the circulating antibody secreting cell response in human diarrhea by a human Lactobacillus strain. Pediatr Res. 1992; 32:141-4. [PubMed: 1324462]

10. Kukkonen K, Nieminen T, Poussa T, Savilahti E, Kuitunen M. Effect of probiotics on vaccine antibody responses in infancy - a randomized placebo-controlled double-blind trial. Pediatr Allergy Immunol. 2006; 17:416-21. [PubMed: 16925686]

11. Sadani DT, Urbaniak SJ, Bruce M, Tighe JE. Repeat ABO-incompatible platelet transfusions leading to hemolytic transfusion reaction. Transfus Med. 2006; 16:375-9. [PubMed: 16999762]

12. AuBuchon JP, de Wildt-Eggen J, Dumont LJ. for the BEST collaborative and the Transfusion Medicine Resource Committee of the CAP. Reducing the variation in performance of antibody titrations. Vox Sang. 2008; 95:57-65. [PubMed: 18479347]

13. Pietersz R, Engelfriet C, Reesink H. Transfusion of apheresis platelets and ABO groups. Vox Sang. 2005; 88:207-21. [PubMed: 15787735]

14. Valbonesi M, De Luigi MC, Lercari G, Florio G, Bruni R, Van Lint MT, Occhini D. Acute intravascular hemolysis in two patients transfused with dry-platelet units obtained from the same ABO incompatible donor. Int J Artif Organs. 2000; 23:642-6. [PubMed: 11059888]

15. Cooling L, Downs T, Butch S, Davenport R. Anti-A and anti-B titers in pooled group O platelets are comparable to apheresis platelets. Transfusion. 2008; 48:2106-13. [PubMed: 18631162]

16. Boothe G, Brecher ME, Root M, Robinson J, Haley R. Acute hemolysis due to passively transfused high-titer anti-B causing spontaneous in vitro agglutination. Immunohematology. 1995; $11: 43-5$. 


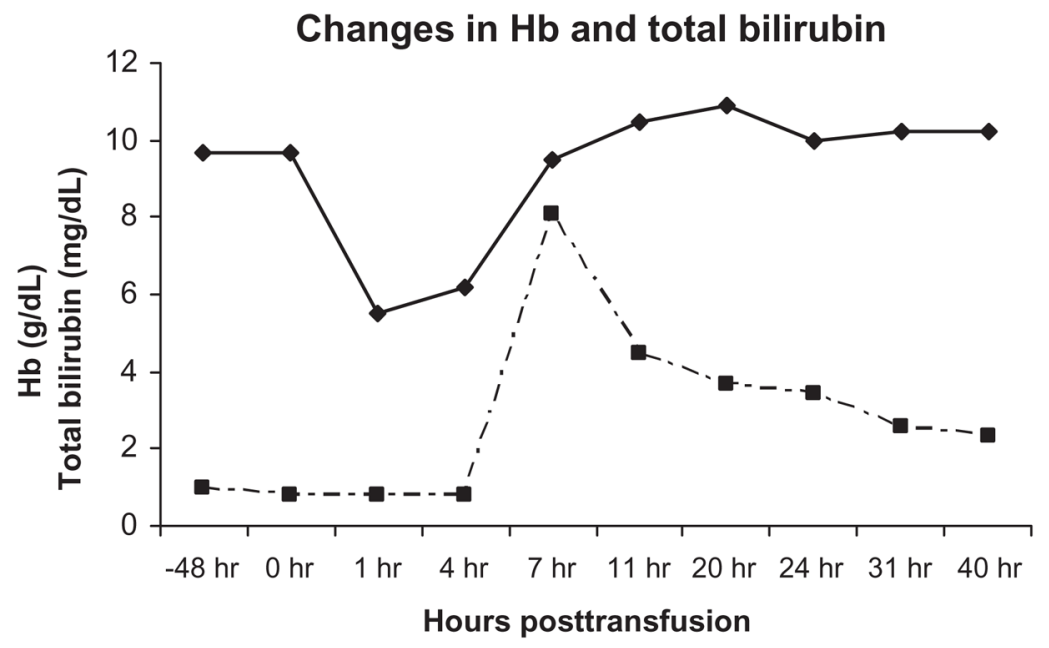

Fig. 1.

Serial $\mathrm{Hb}(>)$ and total bilirubin $(\square)$ values for Case 1. 


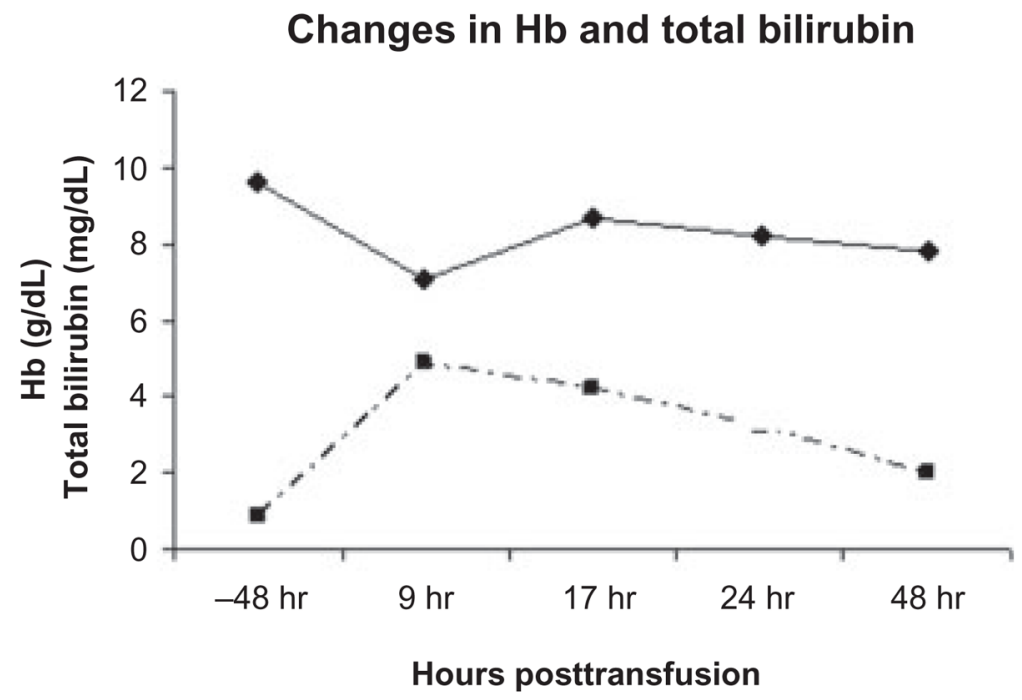

Fig. 2.

Serial $\mathrm{Hb}(>)$ and total bilirubin $(\boldsymbol{\square})$ values for Case 2. 


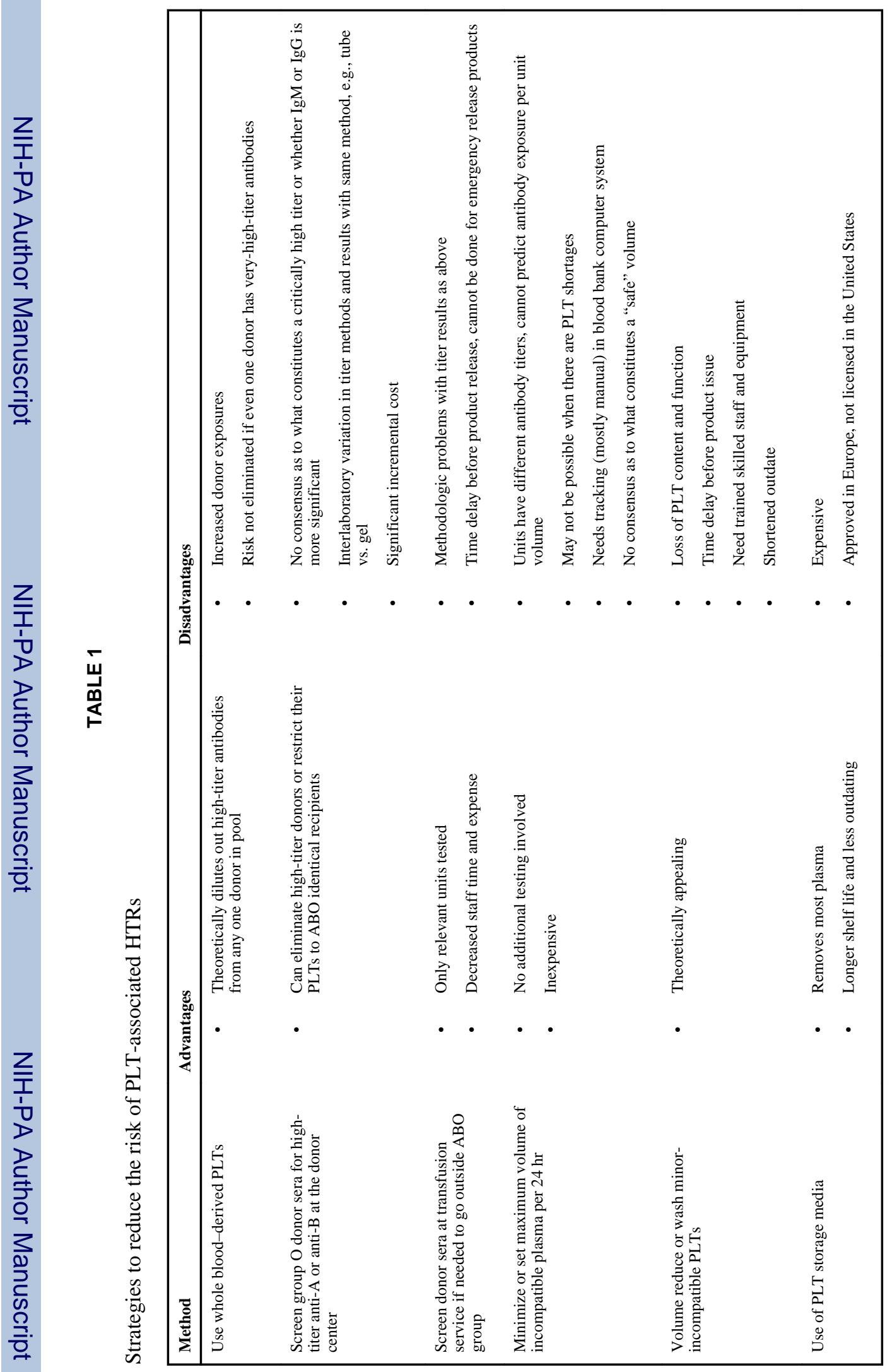

Transfusion. Author manuscript; available in PMC 2012 August 17. 